\title{
Preschool children use space, rather than counting, to infer the numerical magnitude of digits: evidence for a spatial mapping principle.
}

Citation: Sella, F., Berteletti, I., Lucangeli, D., \& Zorzi, M. (2017). Preschool children use space, rather than counting, to infer the numerical magnitude of digits: evidence for a spatial mapping principle. Cognition, (158), 56-67. 


\section{Abstract}

A milestone in numerical development is the acquisition of counting principles which allow children to exactly determine the numerosity of a given set. Moreover, a canonical left-to-right spatial layout for representing numbers also emerges during preschool. These foundational aspects of numerical competence have been extensively studied, but there is sparse knowledge about the interplay between the acquisition of the cardinality principle and spatial mapping of numbers in early numerical development. The present study investigated how these skills concurrently develop before formal schooling. Preschool children were classified according to their performance in Give-a-Number and Number-to-position tasks. Experiment 1 revealed three qualitatively different groups: i) children who did not master the cardinality principle and lacked any consistent spatial mapping for digits, ii) children who mastered the cardinality principle and yet failed in spatial mapping, and iii) children who mastered the cardinality principle and displayed consistent spatial mapping. This suggests that mastery of the cardinality principle does not entail the emergence of spatial mapping. Experiment 2 confirmed the presence of these three developmental stages and investigated their relation with a digit comparison task. Crucially, only children who displayed a consistent spatial mapping of numbers showed the ability to compare digits by numerical magnitude. A congruent (i.e., numerically ordered) positioning of numbers onto a visual line as well as the concept that moving rightwards (in Western cultures) conveys an increase in numerical magnitude mark the mastery of a spatial mapping principle. Children seem to rely on this spatial organization to achieve a full understanding of the magnitude relations between digits.

Keywords: Numerical skills; Counting; Number line; Spatial mapping; Number comparison; Preschool children. 


\section{Highlights:}

1. Counting and spatial mapping of numbers are foundational numerical skills.

2. Cardinal principle knowledge precedes but does not entail consistent spatial mapping.

3. The ability to compare digits by magnitude was linked to spatial mapping but not to cardinal knowledge.

4. The "spatial mapping principle" might be a key to understanding magnitude relationships. 


\section{Introduction}

The development of a symbolic system to represent numerical quantities can be considered one of the most powerful cultural inventions of humans. The shift from an iconic to a symbolic notation has allowed individuals to efficiently denote and manipulate numerical quantities using a variety of transformations (Wiese, 2003), from simple arithmetical operations (i.e., addition and subtraction) to advanced mathematical procedures. From two years of age, young children begin to construct a stable connection between exact numerosities and symbolic representations of numerical quantities. Initially, this mapping is established between number-words and the exact numerosties through the acquisition of the counting principles (Gelman \& Gallistel, 1978). At least three counting principles must be respected to correctly count the items of a given set: (a) the stable-order principle states that the list of number-words must be recited in the correct (received) order (i.e., 1, 2, 3, 4...); (b) the one-to-one correspondence principle claims that each object in the set must be associated with only one number-word in the counting list; and (c) the cardinality principle states that the last recited number-word identifies the number of elements in the set. The correct implementation of these principles allows children to determine the exact numerosity of a given set, thereby creating a meaningful connection between number words and the corresponding objective numerosities. The Give-a-Number (henceforth GaN; Wynn, 1990) is a well-established task to assess the acquisition of cardinality principle in young children. In this task, the experimenter repeatedly asks the child to give a specific number of items drawn from a larger set of objects (e.g., give 3 cookie toys from a basket containing 10 or more cookies). Children's performance shows a stable developmental pattern which follows the acquisition of the cardinal meaning of number-words (c.f. Knower-level theory, Carey, 2001; Sarnecka \& Carey, 2008). At first, children grab a handful of items irrespective of the requested number: These children lack any numerical meaning of number-words and they are referred to as 
pre-number knowers (PN-knowers). Subsequently, children learn the cardinal meaning of the number-word "one" (i.e., one-knowers) and they correctly provide one item when requested.

Interestingly, when requested for a larger numerosity, one-knowers unlikely provide one item because they know the cardinal meaning of the number-word "one". Later, children can correctly give two objects, but they are still unsuccessful with larger numerosities (i.e., two-knowers). With practice, children learn the cardinal meaning of number-words up to four, thereby moving from a PN-knower level to a four-knower level. These children are also denoted as subset-knowers because their cardinal knowledge is limited to a subset from 1 to 4 (Condry \& Spelke, 2008; Le Corre \& Carey, 2007; Le Corre, Van de Walle, Brannon, \& Carey, 2006; Sarnecka \& Gelman, 2004; Wynn, 1990, 1992). Children become cardinal-principle knowers (CP-knowers) when they understand that the next number-word in the count list corresponds to one additional element in the set (i.e., $n+1)$. The CP-knowers extend the cardinal principle to the whole counting list and display a proficient use of counting (Sarnecka \& Carey, 2008). The achievement of the cardinality principle is an effortful process which engages children for approximately over two years, usually from the age of $2-2 \frac{1}{2}$ to $4 \frac{1}{2}-5$ (see Almoammer et al., 2013, for cross cultural variations due to linguistic structure).

Before entering the first year of formal schooling, children have also shown the ability to spatially map $^{1}$ Arabic numbers onto a visual line. In a seminal study, Siegler and Opfer (2003) asked children to place target numbers onto a horizontal line denoting a specific numerical interval (e.g., 0 to 100) by marking its position with a pencil. As demonstrated in many studies that employed this Number-to-Position task, hereafter Number Line (NL) task (Berteletti, Lucangeli, Piazza, Dehaene, \& Zorzi, 2010; Siegler \& Booth, 2004; Siegler \& Lortie-Forgues, 2014; Siegler \& Opfer, 2003), children initially overestimate small numbers and underestimate the position of larger numbers. Progressively,

\footnotetext{
${ }^{1}$ An encyclopaedic definition of mapping is that of "operation that associates each element of a given set with one or more elements of a second set" (e.g., Stevenson \& Lindberg, 2010). In the present paper, we use the term spatial mapping to refer to the association between each number and a spatial position on the visual line (for a more general definition of number-space mapping, see Nunez \& Fias, 2015).
} 
with age and greater familiarity with the numerical interval, children map numbers near the correct location thus showing a linear and accurate positioning. However, accuracy in positioning numbers on a small interval does not grant success on a larger interval. For instance, second graders display an accurate and linear mapping when placing numbers on the 0-100 interval but revert to a biased mapping when placing numbers on the 0-1000 interval (Siegler \& Opfer, 2003). The shift from a biased to an accurate (and formally correct) mapping of numbers has been explained as: (a) the consequence of a shift from a logarithmic to linear representation of numbers (Siegler \& Opfer, 2003); (b) the increasing ability and precision in performing a proportional judgement (Barth \& Paladino, 2011; Slusser, Santiago, \& Barth, 2013); (c) the increased knowledge and familiarity with both the proposed numbers and numerical intervals (Ebersbach, Luwel, Frick, Onghena, \& Verschaffel, 2008; Hurst, Leigh Monahan, Heller, \& Cordes, 2014; Moeller, Pixner, Kaufmann, \& Nuerk, 2009); and (d) the development of measurement skills (Cohen \& Sarnecka, 2014). Regardless of the theoretical interpretation of this finding, the ability to accurately map numbers on the line is strongly correlated with performance in more complex numerical tasks, as well as with mathematical achievement and arithmetic proficiency (Booth \& Siegler, 2008). Crucially, performance in the NL task is a powerful predictor of overall mathematics achievement, which remains reliable even after controlling for arithmetic, reading achievement, and IQ (Booth \& Siegler, 2006, 2008; Sasanguie, Göbel, Moll, Smets, \& Reynvoet, 2013). Moreover, children with math disability display a less accurate performance in the NL task as compared to typical developing children (Geary, Hoard, Nugent, \& Byrd-Craven, 2008; Landerl, Bevan, \& Butterworth, 2004; Sella, Berteletti, Brazzolotto, Lucangeli, \& Zorzi, 2014). Finally, performance in the NL task is correlated with neural activation specific to numerical processing (Berteletti, Man, \& Booth, 2015).

Though the NL task (and its variants) has been extensively used with different numerical and non-numerical intervals (Berteletti, Lucangeli, \& Zorzi, 2012; Sella, Berteletti, Lucangeli, \& Zorzi, 
2015; Siegler, Thompson, \& Opfer, 2009), little is known about the acquisition of the spatial mapping for numbers at early stages of development. In the largest study on preschoolers to date, Berteletti and colleagues (2010) administered the NL task with three numerical intervals (i.e., 1-10, 1-20, 0-100) to pupils belonging to three different age groups (youngest group: $\mathrm{M}_{\text {age }} \approx 4$ y.o.; middle group: $\mathrm{M}_{\text {age }} \approx 5$ y.o.; oldest group: $\mathrm{M}_{\text {age }} \approx 6$ y.o.). In the 1-10 interval, individual mapping analysis highlighted that children shifted from a biased (logarithmic) to an accurate (linear) mapping with age. Nevertheless, a consistent group of children displayed an inconsistent (i.e., not numerically meaningful) mapping throughout the age groups (52\% in the youngest group; $38 \%$ in the middle age group; $15 \%$ in the oldest group). On average, children aged 5 years and above show a linear mapping for the 0/1-10 numerical interval (Berteletti et al., 2010; also see Muldoon, Towse, Simms, Perra, \& Menzies, 2013; Sasanguie, De Smedt, Defever, \& Reynvoet, 2012b; Sasanguie, Van den Bussche, \& Reynvoet, 2012), whereas younger children either show a biased mapping or use a non-numerical strategy to place numbers (e.g., placing numbers in the middle of the line or alternating between right and left side answers irrespective of numerical value). Siegler and Ramani (2008) administered the NL task with the 1-10 interval to preschool children (between 4 and 5 years of age) as a pre-test measure in a training study testing the effectiveness of playing linear board games in enhancing linear numerical representation. In the pre-training analysis, children mainly displayed a non-numerical estimation strategy (e.g., placing numbers in the middle of the line) whereas, after actively playing with a linear board game, children's mapping became linear (see also Ramani, Siegler, \& Hitti, 2012; Siegler \& Ramani, 2009).

As the above studies demonstrate, counting and spatial mapping of numbers are two numerical abilities that emerge already in young preschool children. However, little is known about the relation between these two skills. Young, Marciani and Opfer (2011) found a positive correlation between linearity in the NL task with the 0-20 interval and performance in the GaN task in children between 3 and 5 years of age. Muldoon and colleagues (2013) also found a correlation between linearity in 
different numerical intervals (i.e., 0-10, 0-20,0-100) and counting abilities in a sample of preschool children. Moreover, it has been found that spatial-numerical training enhances children's performance both in the NL task and in counting (Fischer, Moeller, Bientzle, Cress, \& Nuerk, 2011). Berteletti et al. (2010) suggested that the acquisition of the cardinality principle plays a central role in spatial mapping because the ability of preschool children to map numbers linearly was correlated with the ability to numerically order sets of dots. Moreover, in a subsequent study, Berteletti et al. (2012) suggested that linearity is acquired in the numerical domain first and then generalized to other non-numerical ordinal sequences because only numbers possess a cardinal value. All the above studies reported significant correlations between counting and spatial mapping of numbers, however there is sparse knowledge about how these two competences are interrelated in the early stages of development. In particular, previous studies mainly explored general counting skills without focusing on the acquisition of the cardinality principle and how this may eventually interact with the spatial mapping of numbers.

The purpose of the present study was to investigate how the acquisition of cardinality principle and spatial mapping of numbers concurrently develop prior to formal schooling. In particular, we classified preschool children according to their knower-level (using a Bayesian method; Lee \& Sarnecka, 2010) and their ability to spatially map digits in a numerically meaningful way. We examined whether mastery of the cardinality principle (i.e., the CP-knower stage), which marks the achievement of proficient verbal counting, is paralleled by consistent spatial mapping of numbers. If cardinal principle knowledge is acquired before the emergence of spatial mapping, then only CP-knowers should show the ability to consistently map numbers on the visual line. On the other hand, if cardinal principle knowledge entails the ability to map numbers onto the visual line, then all CP-knowers should show consistent spatial mapping. In Experiment 2 we also investigated how the acquisition of cardinality principle and the accuracy in spatial mapping are related to the ability to successfully compare visually presented digits. To the best of our knowledge, these issues have never 
been directly addressed in previous studies. As noted before, a large proportion of the younger preschool children lack any consistent spatial mapping (i.e., whether biased or linear; e.g., Berteletti et al., 2010). Why this occurs has remained largely unexplored. Therefore, it is also conceivable that the cardinality principle is a prerequisite for spatial mapping of numbers per se, which implies that subset-knowers would display an inconsistent (i.e., not numerically meaningful) mapping.

\section{Experiment 1}

In Experiment 1, we assessed children's performance in the GaN and NL tasks. In the GaN, we used a Bayesian classification analysis in order to provide a reliable categorization of children's performance based on the knower-level theory (Lee \& Sarnecka, 2011), whereas for the NL task, children were classified as having a linear, logarithmic or non-numerical mapping following the methodology described in previous research (Berteletti et al., 2010; Siegler \& Opfer, 2003) ${ }^{2}$. To ensure a reliable classification, we assessed children's performance in the NL task three times to obtain a more stable pattern of estimates. Finally, children were asked to recite the number-word list and to name digits to assess their basic numerical knowledge. A large age-range was selected to accurately assess the developmental acquisition of each numerical competence and their interrelation. We expected that only CP-knowers would show a linear mapping of numbers on the line. For subset-knowers, who know the exact meaning of fewer number words, we expected to observe both non-numerical and biased (logarithmic) positioning in the NL task (Berteletti et al., 2010; Siegler \& Ramani, 2009). Alternatively, if mastery of the cardinality principle is a prerequisite for spatial mapping per se, all subset-knowers should not be able to position the numbers in a numerically meaningful (i.e., ordered)

\footnotetext{
${ }^{2}$ Note that the ongoing debate about which model best captures the developmental change in the pattern of estimates in the number-to-position task is orthogonal to the aims of the present study. For this reason, we simply refer to the classic distinction between logarithmic and linear positioning without assuming that the selected model is a faithful index of the underlying representation (also see Berteletti et al., 2012; Sella et al., 2015). Moreover, as further described, the crucial distinction in the present dataset is not between log-like and linear patterns but between consistent (numerically ordered) and inconsistent (i.e., non-numerically meaningful) mapping.
} 
manner.

\section{Material and methods}

\subsection{Participants and Procedure}

Forty-six preschool children (29 boys, $M_{\text {months }}=61, S D=6$, range=48-70) from schools in the north-eastern region of Italy took part in the study after obtaining informed consent from parents or legal guardians. Children were met individually in a separate quiet room during school hours and completed the four tasks in one session. Two fixed orders were chosen to counterbalance the GaN and NL tasks. Half of the participants completed the tasks in the following order: Naming 1-10, NL, Verbal counting and GaN. The other half performed the tasks in the following order: Verbal counting, GaN, Naming 1-10 and NL. There were small breaks between tasks and children could take longer breaks if needed.

\subsection{Numerical Tasks.}

Naming task 1-10. Children were presented with an Arabic digit on a cardboard card (3.7 x 3.7 $\mathrm{cm}$ ) and were asked to name it aloud. All the numbers from 1 to 10 were randomly presented. One point was awarded for each correct naming and the percentage of correct responses was calculated.

Verbal counting task. Children were asked to orally recite the numerical sequence starting from one and were stopped when they reached 20. Children were allowed to correct themselves immediately if they committed an error or to go back and restart the sequence: One point was awarded for each correct number-word and zero for skipped or incorrectly replaced numbers. For instance, if a child skipped the number words from 11 to 14 (included), four errors were counted. The percentage of correct responses was calculated. 
NL task 1-10. A $20 \mathrm{~cm}$ black horizontal line was presented in the middle of a half landscape A4 sheet with the digit one placed just below the left-end of the line and the number ten placed just below the right-end. Children were asked to correctly place a target number on the line by making a mark with a pencil. The number to be positioned was presented inside a box in the upper left corner of the sheet to avoid giving an indication on the midpoint of the line. For every trial, the experimenter said: "This line goes from one to ten [pointing at the numbers]. Where is the correct place for this number [pointing at the number in the upper left corner]? Show me the correct place making a mark with your pencil!". There were two training trials (i.e., 1 and 10 to be placed) in which, if necessary, the experimenter corrected the child by showing the correct position. Then, there were eight randomly presented numbers to be placed (i.e., 2, 3, 4, 5, 6, 7, 8, 9) and no feedback was given. Each child completed this task three times with a brief pause between sessions for a total of 24 test trials.

GaN task. The task was an adaptation of Wynn's $(1990,1992)$ give-a-number. A small basket with fifteen cardboard cards representing oranges ( $7 \mathrm{~cm}$ of diameter) was presented to the child who was free to manipulate them before starting the task. The task was introduced as a role-play game in which the experimenter played the role of a customer and the child played the role of the grocer. The experimenter said: "Let's play the market game! You are a grocer and I'm a customer who wants to buy some delicious oranges. Ok? Are you ready?". The experimenter then said: "Hello! May I have X orange/s, please?". As soon as the child gave the selected number of oranges, the experimenter said: "Is this/Are these X orange/s?". The child was allowed to modify the number of oranges until she was sure about the number. The experimenter asked for 1, 2, 3, 4, 5, 8 and 10 oranges in random order. Each numerosity was asked three times with a brief pause between sessions.

\section{Results}

We analysed the GaN task data in order to categorize children as Subset-knowers or 
CP-knowers. Similarly, we categorized children as displaying linear, logarithmic or non-numerical mapping based on their performance in the NL task. Then, we investigated how the type of spatial mapping was related to the knower level.

GaN analysis. We assigned each child to a knower-level using a Bayesian model (Lee \& Sarnecka, 2010, 2011; Negen, Sarnecka, \& Lee, 2012). The model developed by Lee and Sarnecka (2010) assumes that children's performance in the GaN task can be interpreted as a Bayesian inference based on children's knowledge of numerical concepts and the numerosity requested in each trial by the experimenter (Negen et al., 2012). For instance, if a child knows the meaning of the number-words up to three (i.e., three-knower), the probability of correctly giving items from one to three increases when these numerosities are requested, whereas the probability of producing other numerosities decreases. Conversely, when a three-knower is asked to produce five items, the probability to provide one, two or three elements drastically decreases, whereas the probability of giving other numerosities increases. The most useful application of this model is the possibility to determine each child's knower-level based on her answers in the GaN task. We used the Excel sheet (www.cogsci.uci.edu/codev?negen?Knower-LevelEstimater.xls) developed by Negen and colleagues (2012) to determine the knower-level for each child in our study. Given the large age range in the sample, we set the same prior probabilities for each possible knower-level (i.e., PN-knower, one-knower, two-knower, three-knower, four-knower, and CP-knower). The calculation sheet provides for each child the posterior distribution for each knower-level, that is the probability that a child has a specific knower-level based on the observed answers and the model assumptions (Negen et al., 2012). Therefore, for each child, the knower-level corresponded to the highest peak of her posterior distribution. For example, if a child had a posterior distribution yielding an $82 \%$ probability to be a three-knower and an $18 \%$ to be a four-knower, the child was classified as three-knower. After implementing this procedure, we identified 2 children in the PN-knower, 3 in the one-knower, 3 in the 
two-knower, 2 in the three-knower, 3 in the four-knower, and 33 in the CP-knower groups. Increase in knower-level, with PN-knower classified as 0 to CP-knower classified as 5, was positively correlated with age in months $\left(r_{\mathrm{s}}=.41, p=.005\right)$. In agreement with previous studies (Sarnecka \& Carey, 2008), we considered children ranging from PN-knower to four-knower as Subset-knowers. Therefore, there were 13 Subset-knowers $\left(M_{\text {months }}=57, S D=6\right.$, range=48-69) and 33 CP-knowers $\left(M_{\text {months }}=63, S D=5\right.$, range $=52-70)$.

NL analysis. In order to describe the mapping displayed by children, we ran linear and logarithmic regression analyses on individual median estimates as a function of target numbers. The highest $R^{2}$ determined what type of mapping the child displayed. When neither model was significant, the child was considered unable to properly perform the task and classified as displaying a non-numerical mapping. We also computed the Percentage of Absolute Error using the formula: (|Estimate - Target Number|/Numerical Interval)*100. In agreement with previous studies (Berteletti et al., 2010), we found a positive correlation between the type of mapping (Non-numerical =0; Logarithmic $=1$; Linear $=2)$ and age in months $\left(r_{\mathrm{s}}=0.4, p=.006\right)$.

Intersection between GaN and NL. Central to the purpose of the present study is to investigate the intersection between knower level and type of spatial mapping. All Subset-knowers, except one, displayed a non-numerical mapping of numbers, whereas within CP-Knowers two distinct subgroups were identified, those who mapped numbers (i.e., mappers) either linearly or logarithmically, and those who adopted a non-numerical mapping (i.e., non-mappers; Table 1). We considered a child as a mapper irrespective of whether the best fitting function was linear or logarithmic because both fits entail the understanding of spatial order for numbers with respect to a non-numerical mapping. 
Table 1

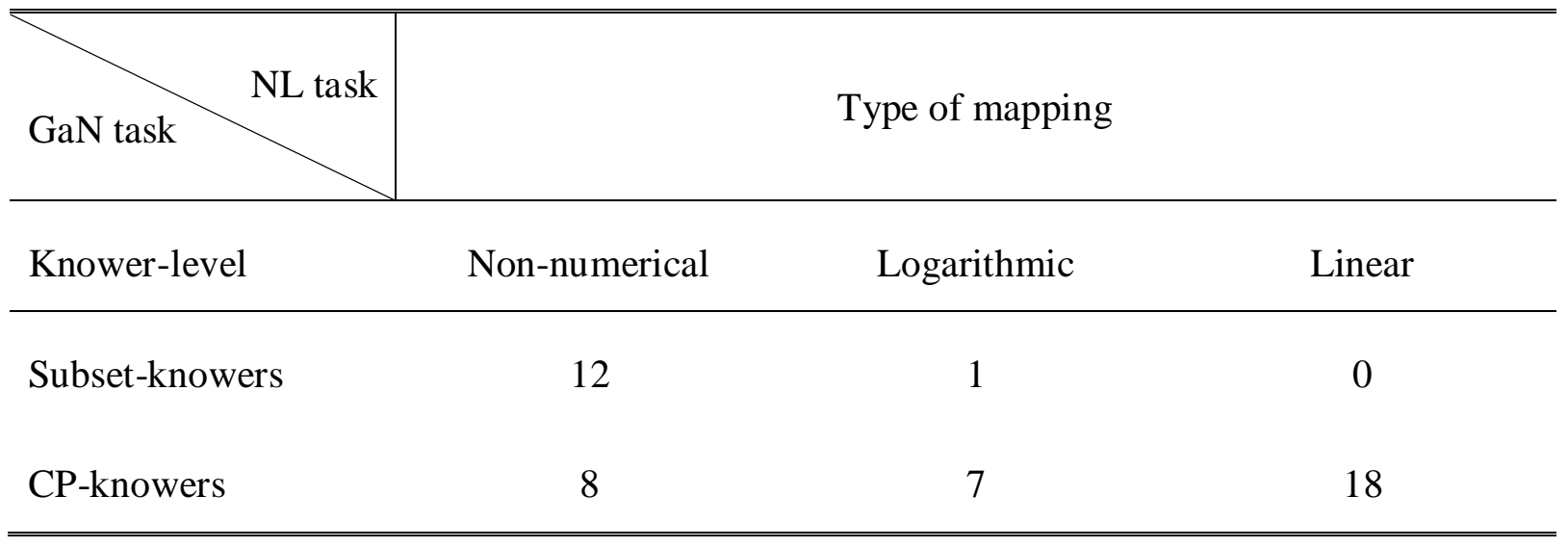

Table 1. Type of mapping in the NL task separated for Subset-knowers and CP-knowers. Cell values represent number of children $(\mathrm{N}=46)$ in Experiment 1.

To further investigate the relation between numerical knowledge and spatial mapping of numbers, we analysed performance in the other tasks subdividing children in three qualitatively different subgroups. Children categorized as subset-knowers in the GaN task and showing a non-numerical mapping in the NL task were defined as Subset-non-mappers $(n=12)$. CP-knowers showing a non-numerical mapping in the NL task were defined as CP-non-mappers $(n=8)$. Finally, CP-knowers displaying a consistent spatial mapping (either linear or logarithmic) in the NL task were defined as CP-mappers $(n=25)$. We fit the linear and logarithmic models on median estimates as a function of target numbers for Susbset-non-mappers, CP-non-mappers and CP-mappers (see Figure 1). Subset-non-mappers and CP-non-mappers displayed a non-numerical mapping (i.e., all estimates in the middle of the line), with both linear and logarithmic models failing to reach significance. CP-mappers, instead, displayed a progressive increase in estimates as a function of number magnitude. Accordingly, the linear model had better fit $\left(R^{2}=.99\right)$ compared to the logarithmic model $\left(R^{2}=.92\right)$, as well as smaller absolute residuals, $t(7)=4.37, p=.003$. 


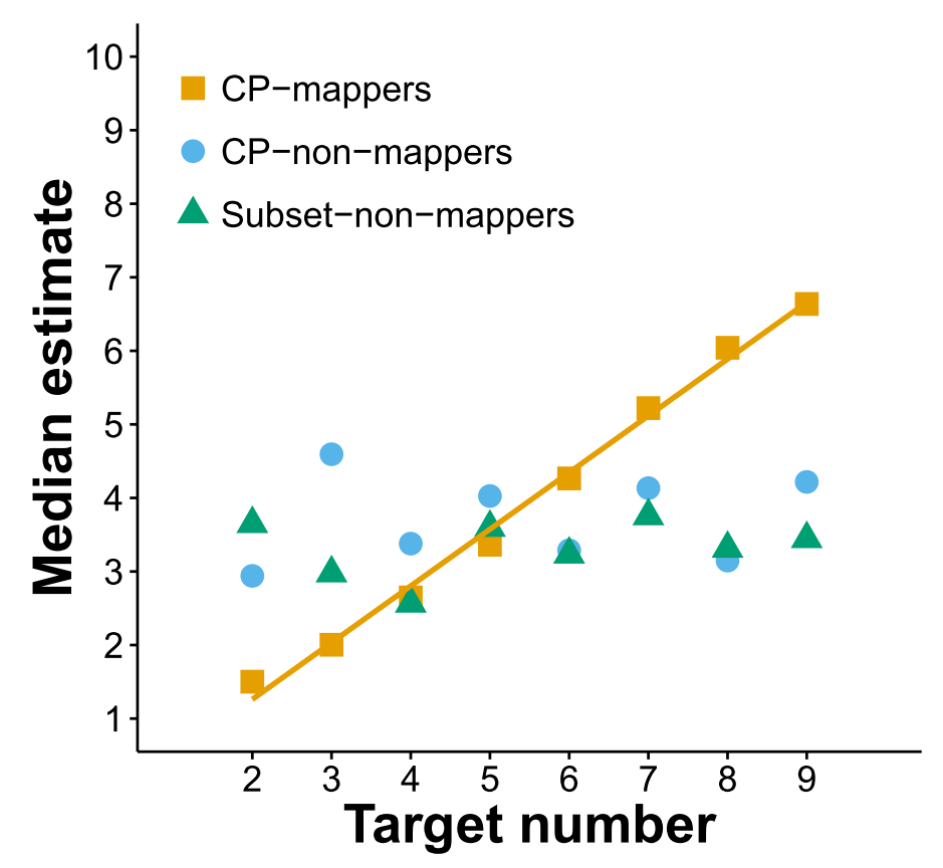

Figure 1. Mean of median estimates of CP-mappers $(\mathrm{N}=25), \mathrm{CP}$-non-mappers $(\mathrm{N}=8)$, and Subset-non-mappers $(\mathrm{N}=12)$ in Experiment 1. The estimates of $\mathrm{CP}$-mappers are best fit by a linear model, while those of the other two groups are unrelated to the magnitude of the target numbers.

To compare the three groups we analysed the percentage of correct responses in the Verbal Counting task and in the Digit Naming task, as well as the percentage of error in the NL task and the age in months, in a series of one-way Kruskall-Wallis tests with Group (Subset-non-mappers, CP-non-mappers, CP-mappers) as between-subjects factor (Field, Miles, \& Field, 2012). Results of main tests and subsequent post-hoc comparisons are reported in Table 2. The Subset-non-mappers had a lower performance in the Naming task compared to the CP-non-mappers. Interestingly, the CP-non-mappers and CP-mappers had similar numerical knowledge (i.e., Verbal Counting and Naming task) and yet CP-non-mappers lacked the ability to meaningfully map numbers onto spatial position for the 1-10 interval. Within the group of CP-mappers, children who displayed a log-like pattern had similar age to those who displayed a linear mapping as well as similar performance in the Verbal Counting and in the Naming tasks (all ps>.05). 
- Insert Table 2 here

Table 2

\begin{tabular}{|c|c|c|c|c|c|c|c|c|c|c|c|c|c|c|}
\hline & \multicolumn{6}{|c|}{ Main analysis } & \multicolumn{8}{|c|}{ Multiple comparisons } \\
\hline Groups & SSnMAP & CPnMAP & CPMAP & & & & $\mathrm{SSnN}$ & $\mathrm{AP}$ vs. & $\mathrm{CPnI}$ & $\mathrm{AP}$ & $\mathrm{CPnN}$ & $\mathrm{AP}$ vs & $\mathrm{CPI}$ & $\mathrm{AP}$ \\
\hline Measures & $\mathrm{M}(\mathrm{SD})$ & $\mathrm{M}(\mathrm{SD})$ & $\mathrm{M}(\mathrm{SD})$ & $\chi^{2}$ & $p$ & $\eta^{2}$ & $Z$ & $p$ & $\mathrm{BC}$ & $R$ & $Z$ & $p$ & $\mathrm{BC}$ & $r$ \\
\hline Verbal counting ( $\%$ correct) & $68(31)$ & $95(14)$ & 93(11) & 10.65 & .005 & 0.24 & -2.35 & .019 & & .37 & -0.80 & .422 & & .10 \\
\hline Naming task $(\%$ correct $)$ & $38(32)$ & $82(23)$ & $93(11)$ & 20.78 & $<.001$ & 0.47 & -2.70 & .007 & $*$ & .43 & -1.22 & .221 & & .15 \\
\hline NL interval 1-10 (PAE) & $36(9)$ & $35(9)$ & $20(10)$ & 17.51 & $<.001$ & 0.40 & -0.34 & .734 & & .05 & -3.22 & .001 & $*$ & .40 \\
\hline Age (months) & $57(6)$ & $60(4)$ & $63(5)$ & 9.46 & .009 & .22 & -1.40 & .161 & & .22 & -1.61 & .108 & & .20 \\
\hline
\end{tabular}

Table 2. Results of statistical analyses comparing Subset-non-mappers (SSnMAP), CP-non-mappers (CPnMAP) and CP-mappers (CPMAP) on numerical tasks and age. Bonferroni correction (BC) adjusted one tail alpha level to .10/8. BC: * $p<.0125$. The effect sizes were calculated as: $\eta^{2}=\chi^{2} / \mathrm{N}-1 ; r=\mathrm{Z} / \sqrt{ } \mathrm{N}$ (Rosenthal, 1991). 


\section{Discussion}

We administered the GaN task and the NL task in order to highlight how cardinal principle knowledge and spatial mapping of numbers are interrelated prior to formal schooling. Children's responses in the GaN task were in line with the knower-level theory which assumes a progressive acquisition of number-words meaning (Sarnecka \& Carey, 2008; Wynn, 1990, 1992). Results in the NL task identified children as displaying a non-numerical (i.e., non-ordered) or a biased logarithmic mapping which tended to become linear with age as observed in previous studies.

Crucially, the intersection between performances in the GaN and NL allowed the identification of three qualitatively different groups: The Subset-non-mappers had the cardinal meaning limited to few number-words and displayed a non-numerical spatial mapping of digits; the CP-non-mappers fully mastered the cardinality principle but displayed a non-numerical (non-ordered) spatial mapping of digits; finally, the CP-mappers fully mastered the cardinality principle and displayed a consistent (either linear or logarithmic) mapping of digits. Interestingly, while subset-knowers performed poorly in digit naming, CP-mappers and CP-non-mappers showed a similar level of numerical knowledge. Therefore, failure in spatial mapping of the latter group does not reflect lack of knowledge of the digits (this issue is further addressed in Experiment 2). These findings suggest that the ability to correctly read Arabic digits along with the mastery of the cardinality principle do not imply a consistent and meaningful spatial mapping of numbers. In a previous study, Le Corre and Carey (2007) also categorized CP-knowers into two qualitatively different groups, those who could reliably estimate rapidly presented discrete quantities larger than four and those who lacked this ability. Interestingly, Le Corre (2014) observed that only the former group could perform correct numerical comparisons (i.e., 10 is larger than 6) thus demonstrating a deeper knowledge of the magnitude relation between numbers words. In this light, the acquisition of counting principles does not directly imply a semantic induction about the magnitude meaning of each number (Davidson, Eng, \& Barner, 2012; Gunderson, Spaepen, 
\& Levine, 2015; Le Corre \& Carey, 2007 but see Odic, Le Corre, \& Halberda, 2015). Given Le Corre and Carey's observation, we questioned whether the ability to spatially map numbers (i.e., digits) was also related to a deeper knowledge of the magnitude relation between digits. This was the aim of the Experiment 2.

\section{Experiment 2}

The aim of Experiment 2 was to replicate the findings of Experiment 1 and extend them by assessing how the acquisition of the cardinality and the accuracy in spatial mapping are related to the ability to perform magnitude comparison of Arabic digits. If the ability to spatially map numbers is related to a deeper understanding of magnitude relations, then children able to position numbers spatially on the line (i.e., CP-mappers), even in a biased (logarithmic) way, should also demonstrate the ability to correctly compare Arabic digits. Conversely, children who lack a consistent spatial mapping of numbers should fail in choosing the larger between two digits despite the acquisition of the cardinality principle and their equivalent knowledge of digits.

In Experiment 2, we administered the same tasks presented in Experiment 1 to a wide agerange of preschool children and added a Digit Comparison task with digits from 1 to 9. We also extended the numerical range tested by evaluating the ability to read numbers between 11 and 20 and to correctly place numbers on a line in the interval between 1 and 20 . These two additional tasks were intended to more thoroughly describe children's numerical skills. Finally, given that in Experiment 1 subset-knowers correctly read on average only four digits out of ten (38\%), in Experiment 2 the numbers to be placed in the NL task were read aloud by the experimenter as to minimize the influence of digit naming skill on performance.

We analysed the GaN task data in order to categorize children as Subset-knowers or 
CP-knowers. Similarly, we categorized children as having a linear, a logarithmic or non-numerical mapping based on their performance to the NL task with 1-10 interval. We expected to observe the same three subgroups as in Experiment 1. Finally, we analysed the performance in the Digit comparison task to test the prediction that the $\mathrm{CP}$-mappers would be the only group showing the ability to correctly compare digits.

\section{Material and methods}

\subsection{Participants and procedure}

Forty-six preschool children ( 28 boys, $M_{\text {months }}=58, S D=11$, range=41-77) from north-eastern Italy took part in the study after obtaining informed consent from parents or legal guardians. Participants completed the same tasks as in Experiment 1 as well as the 1-20 interval for the NL task, a Naming task including numbers from 11 to 20, and a Digit Comparison task. Tasks from Experiment 1 were presented in the same two orders, while the new tasks involving larger numbers were presented last to avoid discouraging children. Presentation orders of the tasks were: a) Naming 1-10, NL task 1-10, Verbal counting 1-20, GaN, Naming 11-20, NL task 1-20 and Digit Comparison or b) Verbal counting, GaN, Naming 1-10, NL 1-10, Digit Comparison, Naming 11-20, and NL task 1-20.

\subsection{Numerical Tasks}

We administered the GaN task, Naming 1-10 task, Verbal Counting task and NL 1-10 task as in Experiment 1 . The only difference was that numbers to position in the NL task were also read aloud to the child (see instructions for the NL task 1-20).

Naming task 11-20. Children were presented with an Arabic digit on a cardboard card (3.7 x 3.7 $\mathrm{cm})$ and were asked to name it aloud. All the numbers from 11 to 20 were randomly presented. One 
point was awarded for each number correctly named and the percentage of correct responses was calculated.

NL task 1-20. A $20 \mathrm{~cm}$ black horizontal line was presented in the middle of a half landscape A4 sheet with the digit one placed just below the left-end of the line, whereas the number twenty was placed just below the right-end. Children were asked to correctly place a number on the line by making a mark with a pencil. The number to be positioned was presented inside a box in the upper left corner of the sheet. For every trial, the experimenter said: "This line goes from one to twenty [pointing at the numbers]. Where is the correct place for this number $X$ [pointing at the number in the upper left corner]? Show me the correct place for X making a mark with your pencil!". There were two training trials (i.e., 1 and 20) in which the experimenter corrected the child if necessary. There were eight randomly presented numbers to position (i.e., 2, 4, 6, 7, 13, 15, 16, and 18). The experimenter read aloud the numbers to be placed. Each child completed the task three times with a brief pause between sessions.

Digit Comparison task. Two single digit numbers, between 1 and 9, were horizontally presented on an A4 landscape sheet (font height was approx. $2.5 \mathrm{~cm}$, the distance between numbers was approx. 9 $\mathrm{cm})$. The child was asked to decide which digit was larger by indicating or naming it. Responses were marked on a record sheet and the child could change her response before moving to the next trial. There were 36 trials presenting all possible comparisons. The larger number was presented equally often in either right or left locations. We calculated the percentage of correct responses as accuracy measure.

\section{Results}

GaN analysis. After the Bayesian classification analysis, we identified 1 PN-knower, 7 one-knowers, 4 two-knowers, 6 three-knowers, 5 four-knowers, and 23 CP-knowers. Therefore, there 
were 23 Subset-knowers $\left(M_{\text {months }}=50, S D=8\right.$, range=41-63) and $23 \mathrm{CP}$-knowers $\left(M_{\text {months }}=66, S D=7\right.$, range $=52-77)$. As observed in Experiment 1, knower-level (from $0=\mathrm{PN}$-knower to $5=\mathrm{CP}$-knower) was positively correlated with age in months $\left(r_{\mathrm{s}}=.76, p<.001\right)$.

NL 1-10 Analysis. As in Experiment 1, we fitted the linear and the logarithmic functions on individual median estimates in order to classify each child as having a linear, logarithmic or non-numerical mapping in the 1-10 interval. We chose to limit the classification to the interval 1-10 for coherence with Experiment 1 and because it represents an interval that should be mastered by children at the end of preschool, thereby yielding greater variability between participants (Berteletti et al., 2010). As observed in Experiment 1, the type of mapping (Non-numerical = 0; Logarithmic $=1$; Linear $=2)$ was positively correlated with age in months $\left(r_{\mathrm{s}}=.53, p<.001\right)$.

Insert Table 3 here

Table 3

\begin{tabular}{l|ccc}
\hline GaN task & & Type of mapping 1-10 interval & \\
\hline Knower-level & Non-numerical & Logarithmic & Linear \\
\hline Subset-knowers & 23 & 1 & 0 \\
CP-knowers & 11 & 3 & 8 \\
\hline \hline
\end{tabular}

Table 3. Type of mapping in the NL task separated for Subset-knowers and CP-knowers. Cell values represent number of children $(\mathrm{N}=46)$ in Experiment 2.

Intersection between GaN and NL. In the 1-10 interval, all Subset-knowers, except one, displayed a non-numerical mapping of numbers onto the line. We classified the CP-knowers in two 
distinct groups, those who mapped numbers (i.e., mappers), whether linearly or logarithmically, and those who displayed a non-numerical mapping (i.e., non-mappers). Based on the type of mapping displayed in the 1-10 interval, we identified 23 Subset-non-mappers, 11 CP-non-mappers, and 11 CP-mappers (see Table 3).

Figure 2
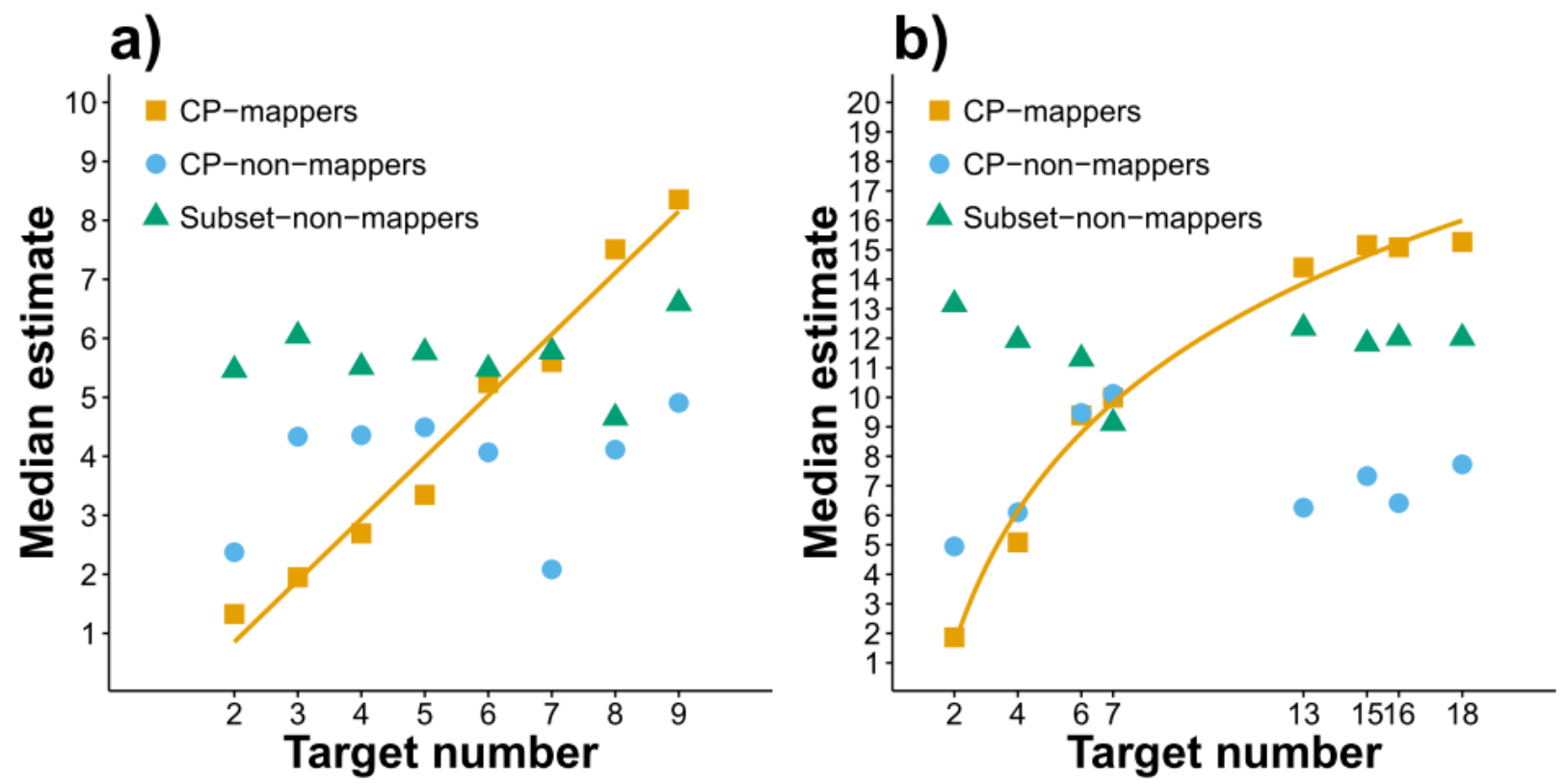

Figure 2. Mean of median estimates for CP-mappers $(\mathrm{N}=11), \mathrm{CP}$-non-mappers $(\mathrm{N}=11)$, and Subset-non-mappers $(\mathrm{N}=23$ ) in the 1-10 interval (panel a) and 1-20 interval (panel b) in Experiment 2. Estimates of CP-mappers are best fit by a linear model in the 1-10 interval and by a logarithmic model in the 1-20 interval. The estimates of the other two groups are unrelated to the magnitude of the target numbers.

We fit the linear and logarithmic models on median estimates as a function of target numbers for Subset-non-mappers, CP-non-mappers and CP-mappers separately for the 1-10 and 1-20 intervals (see Figure 2). In both numerical intervals, Subset-non-mappers and CP-non-mappers displayed a non-numerical mapping (i.e., all estimates in the middle of the line), with both linear and logarithmic 
models failing to reach significance. CP-mappers, instead, displayed a progressive increase in estimates as a function of the magnitude of target numbers. For the 1-10 interval, the linear model had a better fit $\left(R^{2}=.98\right)$ compared to the logarithmic model $\left(R^{2}=.89\right)$, as well as smaller absolute residuals, $t(7)=3.49$, $p=.01$. Conversely, the logarithmic model had a better fit $\left(\mathrm{R}^{2}=.98\right)$ compared to the linear model $\left(R^{2}=.89\right)$, as well as smaller absolute residuals, $t(7)=3.49, p=.01$, for the $1-20$ interval.

For Verbal Counting, Naming and Digit Comparison tasks we calculated the percentage of correct responses, whereas for the two NL tasks we calculated the percentage of error with the same formula as in Experiment 1. These variables were then analysed, along with age in months, in a series of one-way Kruskall-Wallis tests with Group [Subset-non-mappers, CP-non-mappers, CP-mappers] as between-subjects factor to compare the three groups. Main results and subsequent post-hoc comparisons are reported in Table 4. Subset-non-mappers displayed a lower performance in Verbal Counting and in 1-10 Naming; they were also significantly younger compared to the CP-non-mappers. CP-non-mappers and CP-mappers had similar numerical knowledge (i.e., Verbal Counting and Naming scores) and age. $\mathrm{CP}$-mappers, compared to $\mathrm{CP}$-non-mappers, showed better performance in both the NL tasks and importantly they showed greater accuracy in the Digit Comparison task (Figure 3). Interestingly, within the group of CP-mappers, accuracy in the Digit Comparison task was very similar for children displaying a $\log$-like pattern $(M=95 \%, S D=6)$ and children displaying a linear pattern $(M=96 \%, S D=5)^{3}$.

\footnotetext{
${ }^{3}$ For all CP-mappers, we also estimated the individual logarithmic component (i.e., $\lambda$ ) using a mixed log-linear model (Anobile, Cicchini, \& Burr, 2012; Opfer, Thompson, \& Kim, 2016). The $\lambda$ parameter estimation is constrained to assume values between 0 (linear model) to 1 (logarithmic model). CP-mappers with a log-like mapping pattern displayed a slightly positive and larger lambda $(M=0.37, S D=0.28)$ compared to $C P$-mappers with a linear mapping pattern who displayed a lambda equal to zero $(M=0, S D=0)$. We also computed the correlation between lambda and digit comparison accuracy, which should be taken as explorative due to the very small sample size. The correlation was modest and it failed to reach significance $(r=-.37, t(9)=1.19, p=.27)$..
} 


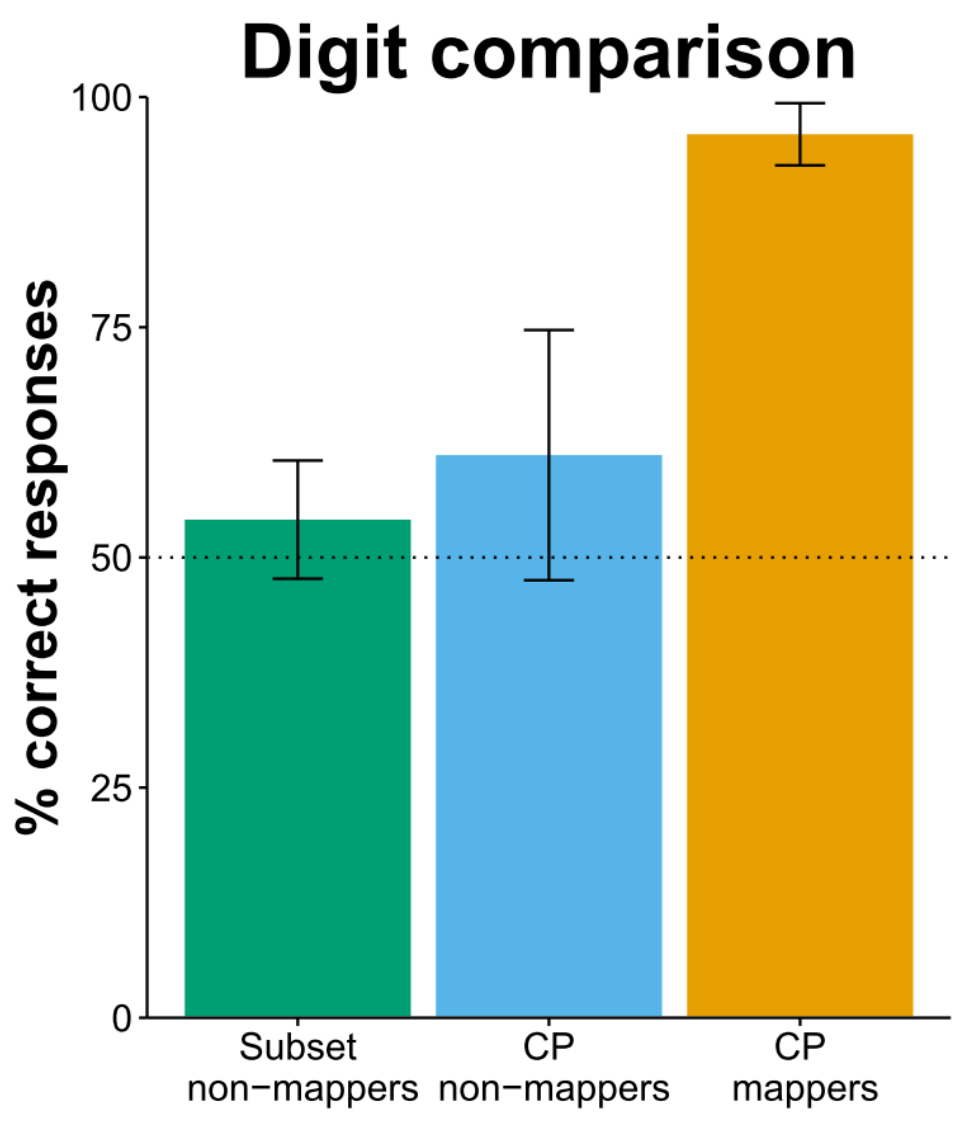

Figure 3. Percentage of correct responses in the digit comparison task for Subset-non-mappers, CP-non-mappers and CP-mappers. Bars represent the 95\% CI and the dotted line represents the chance level (i.e., $50 \%$ of correct responses).

We further explored whether the ability to compare digits in $\mathrm{CP}$-mappers and $\mathrm{CP}$-non-mappers might be modulated by number magnitude. In this regard, Le Corre (2014) found that only a subgroup of CP-knowers who could reliably estimate visually presented objects ranging from 6 to 10 were also able to tell that "ten" is more than "six" and more than "eight". Nevertheless, all CP-knowers could reliably say that "three" is more than "two" and that "eight" is more than "one", thereby suggesting that they could successfully compare small to large digits but not large to large digits. In fact, before becoming CP-knowers, children learn the meaning of number-words up to four and know that all number words beyond three denote larger numerosities (Condry \& Spelke, 2008; Le Corre \& Carey, 
2007; Wynn, 1992). Accordingly, we analysed the response accuracy for comparisons that include one digit smaller than or equal to 4 separately from comparisons that only include digits greater than 4 (there were ten trials of the latter type: 5-6, 5-7, 8-5, 9-5, 7-6, 6-8, 6-9, 8-7, 7-9, 8-9). We analysed the mean percentage of correct responses in a mixed ANOVA with Type of Comparison [Small, Large] as within-subjects factor and Group [CP-mappers, CP-non-mappers] as between-subjects factor. The main effects of Type of Comparison, $F(1,20)=10.05, \mathrm{MSE}=107.9, p=.005, \eta_{\mathrm{p}}{ }^{2}=.33$, and Group, $F(1$, $20)=25.63, \mathrm{MSE}=477.5, p<.001, \eta_{\mathrm{p}}{ }^{2}=.56$, were both significant whereas the interaction Type of Comparison $x$ Group failed to reach significance, $F(1,20)=1.15, \mathrm{MSE}=107.9, p=.297, \eta_{\mathrm{p}}{ }^{2}=.05$. Accuracy was slightly higher for small than for large comparisons across groups, but this did not reduce the large performance gap between CP-mappers (Small: $M=99.6 \%, S D=1 \%$; Large: $M=86 \%$, $S D=16 \%$ ) and CP-non-mappers (Small: $M=63 \%, S D=21 \%$; Large: $M=56 \%, S D=22 \%$ ).

Strikingly, performance of CP-non-mappers was at chance level for the comparison of large numbers (one-tailed t-test vs. 50\%: $t(10)=0.98, \mathrm{p}=.18$ ). Their performance was above chance for comparisons involving at least one small number $(t(10)=2.05, \mathrm{p}=.034)$, but it reverted to chance level when trials involving pairs of small numbers (e.g., 2-4) were excluded ( $M=60.9 \%, S D=24 \%$; $t(10)=1.49, p=.0 .08)$. Overall, the ability of CP-non-mappers to compare visually presented Arabic digits was extremely poor, in sharp contrast with the mature ability displayed by CP-mappers. 
Table 4

\begin{tabular}{|c|c|c|c|c|c|c|c|c|c|c|c|c|c|c|}
\hline & \multicolumn{6}{|c|}{ Main analysis } & \multicolumn{8}{|c|}{ Multiple comparisons } \\
\hline Groups & SSnMAP & CPnMAP & CPMAP & & & & SSnN & AP vs. & $\overline{\mathrm{CPnN}}$ & IAP & $\mathrm{CPnI}$ & IAP vs. & CPN & $\overline{\mathrm{AP}}$ \\
\hline Verbal counting (\% correct) & $51(30)$ & $86(18)$ & $93(13)$ & 18.62 & $<.001$ & .42 & -3.18 & .001 & $*$ & .39 & -0.93 & .35 & & .14 \\
\hline $\begin{array}{l}\text { Naming task 11-20 (\% } \\
\text { correct) }\end{array}$ & $5(7)$ & $12(24)$ & $38(40)$ & 9.93 & .007 & .23 & -0.64 & .524 & & .08 & -2.04 & .041 & & .31 \\
\hline Digit comparison (\% correct) & $54(15)$ & $61(20)$ & $96(5)$ & 20.85 & $<.001$ & .47 & -0.98 & .325 & & .12 & -3.54 & $<.001$ & $*$ & .53 \\
\hline Age (months) & $50(8)$ & $63(7)$ & $69(6)$ & 26.31 & $<.001$ & .60 & -3.71 & $<.001$ & $*$ & .45 & -1.91 & .056 & & .29 \\
\hline
\end{tabular}

Table 4. Results of statistical analyses comparing Subset-non-mappers (SSnMAP), CP-non-mappers (CPnMAP) and CP-mappers (CPMAP) on numerical tasks and age. Bonferroni correction (BC) adjusted one tail alpha level to .10/14. BC: * $p<.006$. The effect sizes were calculated as: $\eta^{2}=\chi^{2} / \mathrm{N}-1 ; r=\mathrm{Z} / \sqrt{ } \mathrm{N}($ Rosenthal, 1991). 
In order to highlight the different contribution of cardinal principle knowledge and spatial mapping of numbers on the ability to compare digits, we ran two hierarchical regression analyses with percentage of correct responses in the Digit Comparison task as outcome variable. Indeed, within the CP-knowers group, different levels of performance could potentially relate to differences in magnitude comparison skill. Therefore, to account for within group variability in counting proficiency, we used a continuous measure (percentage of correct responses). In both models, age in months was entered as first predictor, which explained a large amount of variance (see Table 5), followed by accuracy in Verbal Counting and Naming. For the first model (see Model A in Table 5), we added the percentage of correct responses in the GaN task, which did not explain additional variance (Step 3A), and percentage of error in the NL task, which explained a significant and consistent amount of variance (Step 4A). In the second model (see Model B in Table 5), we first added the percentage of error in the NL task with the interval 1-10 (Step 3B), which improved significantly the variance explained, whereas entering the percentage of correct responses in the GaN task as last predictor failed to increase the fit of the model (Step $4 B$ ). The two regression models suggest that the ability to compare digits is related to the spatial mapping of numbers rather than to the mastery of the cardinality principle, even when the effects of age and basic numerical knowledge (i.e., Verbal Counting and Naming) were statistically removed. 
Table 5

\begin{tabular}{|c|c|c|c|c|c|c|c|c|c|c|c|}
\hline Model A & & & & & & Model B & & & & & \\
\hline Constant & & -0.163 & 0.136 & & .238 & Constant & & -0.163 & 0.136 & & .238 \\
\hline Step 2 & .051 & & & & & Step 2 & .051 & & & & \\
\hline Constant & & -0.007 & 0.162 & & .967 & Constant & & -0.007 & 0.162 & & .967 \\
\hline Age (months) & & 0.009 & 0.003 & $0.424 *$ & .014 & Age (months) & & 0.009 & 0.003 & $0.424 *$ & .014 \\
\hline Naming task $1-10$ (\% correct) & & 0.121 & 0.091 & 0.224 & .193 & Naming task $1-10$ (\% correct $)$ & & 0.121 & 0.091 & 0.224 & .193 \\
\hline Constant & & -0.009 & 0.164 & & .957 & Constant & & 0.739 & 0.213 & & .001 \\
\hline Age (months) & & 0.009 & 0.004 & $0.448 *$ & .021 & Age (months) & & 0.002 & 0.003 & 0.094 & .547 \\
\hline Naming task $1-10$ (\% correct) & & 0.147 & 0.127 & 0.271 & .256 & Naming task $1-10$ (\% correct $)$ & & 0.120 & 0.076 & 0.221 & .122 \\
\hline Verbal counting (\% correct) & & 0.125 & 0.108 & 0.168 & .256 & Verbal counting (\% correct) & & 0.091 & 0.085 & 0.123 & .290 \\
\hline $\mathrm{GaN}(\%$ correct $)$ & & -0.001 & 0.002 & -0.082 & .771 & NL 1-10 (PAE) & & -0.008 & 0.002 & $-0.535^{* * *}$ & $<.001$ \\
\hline Step 4A & .166 & & & & & Step 4B & .005 & & & & \\
\hline $\mathrm{GaN}(\%$ correct $)$ & & -0.001 & 0.002 & -0.178 & .448 & $\mathrm{GaN}(\%$ correct $)$ & & -0.001 & 0.002 & -0.178 & .448 \\
\hline NL 1-10 (PAE) & & -0.009 & 0.002 & $-0.543 * * *$ & $<.001$ & NL 1-10 (PAE) & & -0.009 & 0.002 & $-0.543 * * *$ & $<.001$ \\
\hline
\end{tabular}

Table 5. Hierarchical regression models with percentage of correct responses in the Digit Comparison task as outcome measure. In both models, Age was entered as predictor in Step 1, whereas numerical knowledge (i.e., percentage of correct responses in the Naming and in the Verbal Counting task) was entered in Step 2. In Model A, percentage of correct responses in the GaN task was entered in Step 3A and Percentage of Absolute Error (PAE) in the 1-10 NL task was entered in Step 4A. Conversely, in the Model B PAE in the NL was entered in Step 3B and accuracy in the GaN task was entered in Step 4B. Regardless of the order in which it was inserted as predictor, GaN did not significantly improve the model fit. 


\section{Discussion}

In Experiment 2, we replicated the observation that the intersection between cardinal principle knowledge and spatial mapping of numbers yields three qualitatively different groups. The three groups also showed qualitative differences in basic numerical skills: Subset-non-mappers displayed a reduced ability in naming digits from 1 to 10 as well as a reduced performance in reciting the verbal counting sequence up to 20 compared to CP-non-mappers. These two groups also differed in terms of age, with the Subset-non-mappers being younger compared to the $\mathrm{CP}$-non-mappers. Crucially, $\mathrm{CP}$-mappers outperformed CP-non-mappers in the Digit Comparison task despite the similar basic numerical knowledge. Unsurprisingly, CP-mappers showed lower error in positioning numbers on both number line intervals (i.e., 1-10 and 1-20). The hierarchical regression showed that, after controlling for age and basic numerical knowledge, the performance in comparing digits is explained by the precision in spatially mapping numbers rather than proficiency in counting. Indeed, only children who displayed a consistent spatial mapping of numbers (i.e., $\mathrm{CP}$-mappers) were able to accurately select the larger between two visually presented digits. Similarly to the results of Le Corre et al. (Le Corre, 2014; Le Corre \& Carey, 2007), the mastery of the cardinality principle did not imply understanding of the magnitude relation between numbers.

\section{General discussion}

The present study investigated the relation between the acquisition of the cardinality principle and spatial mapping of numbers in preschool children. We classified children as Subset-knowers or CP-knowers using a robust Bayesian analysis of the GaN task performance (Lee \& Sarnecka, 2011; Negen et al., 2012). In line with previous studies, the individual knower-level strongly correlated with age thus suggesting that the progressive acquisition of cardinal knowledge follows a specific developmental trajectory (Sarnecka \& Carey, 2008; Wynn, 1992). For the spatial mapping, in both 
experiments we repeatedly asked children to position the target numbers on the visual line with the 1-10 interval in order to classify their mapping as non-numerical, biased (logarithmic), or linear. As in previous studies, the precision in estimating numbers on the line was correlated with age (Berteletti et al., 2010; Muldoon et al., 2013; Sasanguie, De Smedt, et al., 2012; Sasanguie, Van den Bussche, et al., 2012; Siegler \& Opfer, 2003; Young et al., 2011).

As central purpose of this study, we categorized children according to their performance in both the GaN and NL tasks to obtain a clear picture of the interplay between the acquisition of the cardinality principle and the spatial mapping of numbers. The two experiments highlighted the presence of three developmental stages: Subset-non-mappers lacked the understanding of the cardinality principle and placed numbers on the visual line with a mapping that was not numerically meaningful (i.e., non-ordered). Importantly, we excluded that non-numerical mapping in the NL task could be due to poor number reading because the numbers to be placed on the line were named aloud by the experimenter (Experiment 2). A second group, defined as CP-non-mappers, showed the mastery of the cardinality principle but displayed a non-numerical mapping of numbers. Notably, these children also showed poor performance in choosing the larger between two visually presented digits. Indeed, they failed when comparing large digits and performed slightly above the chance level only when asked to compare pairs of digits which included at least a small number (i.e., $\leq 4)$. Finally, the CP-mappers mastered the cardinality principle, displayed a consistent spatial mapping of numbers, and also successfully chose the larger between two visually presented digits.

The existence of the CP-non-mappers group, replicated across the two experiments, suggests that mastery of the cardinality principle does not entail the ability to map numbers on the visual line in a consistent way, as well as to reliably determine the larger between two digits. It is worth to emphasize that in the GaN task, CP-non-mappers reliably associated each number-word to the corresponding exact numerosity. Nevertheless, CP-non-mappers had a poor performance in the digit comparison task where 
the magnitude (semantic) relation between two digits needs to be accessed. Importantly, this failure cannot be attributed to poor knowledge of the symbolic numbers given that they showed the same performance of CP-mappers in the Digit Naming task 1-10 (95\% vs 96\% accuracy). The finding that $\mathrm{CP}$-non-mappers had a poor digit comparison performance is consistent with previous observations that some CP-knowers may fail in choosing the larger between two numbers presented as number-words (e.g., "ten" and "eight"; Le Corre, 2014), or in adding 1 to a given number-word (Davidson et al., 2012). Some CP-knowers may also fail to provide reasonable verbal estimates for the numerosity of a set without counting (Gunderson et al., 2015; Le Corre \& Carey, 2007).

Le Corre (2014) found that only a subgroup of CP-knowers could verbally estimate the numerosity of sets larger than 4. Interestingly, only this subgroup of CP-knowers was also able to compare two verbally presented digits when both were larger than 4, suggesting that the cardinality principle per se does not imply that number-words have been systematically mapped on internal numerical representations (Le Corre, 2014). The finding that some CP-knowers fail in verbal numerosity estimation has been recently accounted for in terms of asymmetry in the development of bidirectional mappings between number-words and ANS, with the ANS to number-words mapping established later than the opposite mapping (Odic et al., 2015). Indeed, all CP-knowers, including those with poor verbal numerosity estimation, were able to reliably generate an approximate number by rapid tapping in response to a verbal number (Odic et al., 2015). This suggests that, at least in principle, children could use the earlier-acquired mapping from number-words to ANS: that is, reading the target digits should activate the corresponding non-symbolic representations and allow a magnitude comparison. However, this was clearly not the case for our CP-non-mappers, whose performance in number comparison remained poor even for trials involving one small digit. Overall, our findings suggest that the ability to compare the full range of one-digit numbers is not strictly linked to the development of mappings between symbols and ANS. Indeed, leading computational accounts of 
symbolic number comparison (see Verguts, Fias, \& Stevens, 2005; Zorzi \& Butterworth, 1999; Zorzi, Stoianov, \& Umiltà, 2005) assume a semantic representation of exact numbers that encodes magnitude relationships but is distinct from the ANS. Most importantly, our results show that proficient digit comparison is achieved at a developmental stage that is characterized by the emergence of consistent spatial mapping of numbers.

We propose that congruent (i.e., numerically ordered) positioning of numbers onto a visual line as well as the concept that moving rightwards (in Western cultures) conveys an increase in numerical magnitude mark the mastery of a spatial mapping principle. This implies reliance on a layout composed of spatially ordered numbers in which moving rightwards on a line, whether real or imaginary (Zorzi, Priftis, \& Umiltà, 2002), conveys an increase in numerical magnitude (note, however, that the spatial direction corresponding to magnitude increments is culture-dependent; Gobel, Shaki, \& Fischer, 2011, for review). This spatial mapping principle might guide children in understanding the magnitude relations between digits, thereby allowing a successful accomplishment of the digits comparison task. In line with our results, Opfer, Thompson, and Furlong (2010), observed that preschool children with a clear left-to-right association between numbers and space also demonstrated more mature numerical skills.

The link between spatial mapping and the understanding of magnitude relationships is consistent with the hypothesis that the representation of numerical magnitude is grounded in space (Hubbard, Piazza, Pinel, \& Dehaene, 2005; Zorzi et al., 2002) and that digit comparison involves shifts of spatial attention on the mental number line (Ranzini et al., 2014; Ranzini, Lisi, \& Zorzi, 2016; Stoianov, Kramer, Umiltà, \& Zorzi, 2008; Zorzi et al., 2012). This fits the proposal that a number-space mapping is already present in infants in the form of spontaneous association between numerosity and spatial extent (de Hevia \& Spelke, 2010; Lourenco \& Longo, 2010), or in the form left/small-right/large associations for non-symbolic numerical quantities (Patro \& Haman, 2012). Space 
is an excellent frame of reference for mapping a variety of magnitudes/quantities (from numbers to non-spatial continuous quantities, Sella, Berteletti, Lucangeli, \& Zorzi, 2015; also see Möhring, Ramsook, Hirsh-Pasek, Golinkoff, \& Newcombe, 2016, for musical pitch) as well as non-numerical order (Berteletti, Lucangeli, \& Zorzi, 2012; Zorzi, Priftis, Meneghello, Marenzi \& Umiltà, 2006) and time (Bonato, Zorzi, \& Umiltá, 2012, for review). Nevertheless, our results also show that the initial development of counting does not require spatial mapping of numbers. Indeed, CP-non-mappers were able to proficiently count and read numbers even if they could not map them onto space.

One potential caveat is that the difference between CP-mappers and CP-non-mappers might be related to domain-general factors. For example, children who show an earlier acquisition of the spatial mapping principle may possess better visuospatial skills (Sella, Sader, Lolliot, \& Cohen Kadosh, 2016; Simms, Clayton, Cragg, Gilmore, \& Johnson, 2016; Thompson, Nuerk, Moeller, \& Cohen Kadosh, 2013). Alternatively, CP-mappers might be children who tend to spontaneously focus on numerical information (Hannula \& Lehtinen, 2005; Hannula, Lepola, \& Lehtinen, 2010; Sella, Berteletti, Lucangeli, \& Zorzi, 2015a) and, as a consequence, have more experience with numbers and better awareness of their spatial ordering in daily environments (see McCrink \& Opfer, 2014 for a review on the development of spatial-numerical association). These issues could be addressed in future studies.

In a broader perspective, the ability to map numbers to other dimensions should be considered a milestone in the acquisition of basic numerical competences.

Preschool children display the ability to associate discrete numerosities (e.g., sets of dots) to verbal labels (Barth, Starr, \& Sullivan, 2009; Odic, Le Corre, \& Halberda, 2015; Sullivan \& Barner, 2014) and the precision of mapping demonstrates a significant correlation with numerical and mathematical knowledge in advanced stages of development (Brankaer, Ghesquière, \& De Smedt, 2014; Castronovo \& Göbel, 2012). Similarly, the ability to map symbolic quantities onto spatial positions on the line has also been found to correlate with math achievement (Booth \& Siegler, 2006, 2008; Sasanguie, De 
Smedt, et al., 2012) and to be compromised in children with math disability (Geary et al., 2008;

Landerl et al., 2004; Sella et al., 2014). A crucial question is whether these association skills precede or follow the understanding of magnitude relations between numbers. Indeed, children are likely to use the information conveyed by these associations (spatial or verbal) to infer magnitude relations between numbers (e.g., the numbers get larger moving rightward on the line or stepping forward through the counting list). However, it is also conceivable that some children learn magnitude relations in a trial-and-error process under the supervision of adults (or more skilled peers) and then understand that the larger numbers should be placed on the rightmost part of the line or that the later number-word in the counting list denotes a larger numerosity. Both scenarios are plausible and they are not mutually exclusive if we assume that children exploit all the information at their disposal to understand the semantic knowledge conveyed by symbolic numbers, which represent a key component for future math achievement (Merkley \& Ansari, 2016).

\section{Conclusion}

In the present study, the classification of children's performance in the GaN and the NL tasks revealed the presence of three distinct developmental stages in the acquisition of the cardinality principle and spatial mapping of numbers. In the first stage, children have limited knowledge of symbolic numbers, do not master the cardinality principle, and fail to show a numerically meaningful spatial mapping of numbers. Subsequently, children improve their knowledge of numbers, they can name single digits accurately, and show the mastery of the cardinality principle; however, they still fail in mapping numbers onto space in a meaningful way and show a poor performance in choosing the larger between two digits. Finally, children show the ability to meaningfully translate numbers into spatial positions on a line and can also compare numbers based on their magnitude. Therefore, spatial 
mapping of numbers might be the key to fully understand the magnitude relations between numbers. The latter hypothesis fits well with the finding that performance of skilled adults in symbolic number comparison is influenced by experimental manipulations of spatial-attentional processing (Kramer, Stoianov, Umiltà, \& Zorzi, 2011; Ranzini et al., 2014; Stoianov, Kramer, Umiltà, \& Zorzi, 2008; also see Cutini, Scarpa, Scatturin, Dell'Acqua, \& Zorzi, 2014 for recent neuroimaging evidence of number-space interactions at the semantic level of representation) as well as by impaired spatial processing after brain damage (Vuilleumier, Ortigue, \& Brugger, 2004; Zorzi et al., 2012). More generally, it is conceivable that the acquisition of spatial mapping marks the emergence of a mental representation of numbers that is characterized by a spatial metric similar to that of a (real or imagined) visual line (Zorzi et al., 2002).

In conclusion, congruent (i.e., numerically ordered) positioning of numbers onto a visual line as well as the concept that moving rightwards (in Western cultures) conveys an increase in numerical magnitude mark the mastery of a spatial mapping principle. Children seem to rely on this spatial organization to achieve a full understanding of the magnitude relations between digits. 
Acknowledgments: This study was supported by the University of Padova (Strategic Grant "NEURAT" to M.Z.). The Authors wish to thank the children and their parents for participating in the study, as well as Anna Cappellari, Alice Pasqualotto, and Roberta Zamprogno for their help in collecting data. The Authors are grateful to two anonymous reviewers for their helpful and constructive comments, which helped us to improve the manuscript. 


\section{References}

Almoammer, A., Sullivan, J., Donlan, C., Marušič, F., Žaucer, R., O’Donnell, T., \& Barner, D. (2013). Grammatical morphology as a source of early number word meanings. Proceedings of the National Academy of Sciences of the United States of America, 110(46), 18448-53. http://doi.org/10.1073/pnas.1313652110

Barth, H. C., \& Paladino, A. M. (2011). The development of numerical estimation: evidence against a representational shift. Developmental Science, 14(1), 125-35. article. http://doi.org/10.1111/j.1467-7687.2010.00962.x

Barth, H. C., Starr, A., \& Sullivan, J. (2009). Children's mappings of large number words to numerosities. Cognitive Development, 24(3), 248-264. article. http://doi.org/10.1016/j.cogdev.2009.04.001

Berteletti, I., Lucangeli, D., Piazza, M., Dehaene, S., \& Zorzi, M. (2010). Numerical estimation in preschoolers. Developmental Psychology, 46(2), 545-51. article. http://doi.org/10.1037/a0017887

Berteletti, I., Lucangeli, D., \& Zorzi, M. (2012). Representation of numerical and non-numerical order in children. Cognition, 124(3), 304-313. article. http://doi.org/10.1016/j.cognition.2012.05.015

Berteletti, I., Man, G., \& Booth, J. R. (2015). How number line estimation skills relate to neural activations in single digit subtraction problems. NeuroImage, 107, 198-206. http://doi.org/10.1016/j.neuroimage.2014.12.011

Bonato, M., Zorzi, M., \& Umiltá, C. (2012). When time is space: Evidence for a mental time line. Neuroscience and Biobehavioral Reviews, 36(10), 2257-2273. http://doi.org/10.1016/j.neubiorev.2012.08.007

Booth, J. L., \& Siegler, R. S. (2006). Developmental and individual differences in pure numerical 
estimation. Developmental Psychology, 42(1), 189-201. article.

http://doi.org/10.1037/0012-1649.41.6.189

Booth, J. L., \& Siegler, R. S. (2008). Numerical magnitude representations influence arithmetic learning. Child Development, 79(4), 1016-31. article. http://doi.org/10.1111/j.1467-8624.2008.01173.x

Brankaer, C., Ghesquière, P., \& De Smedt, B. (2014). Children's mapping between non-symbolic and symbolic numerical magnitudes and its association with timed and untimed tests of mathematics achievement. PLoS ONE, 9(4), e93565. http://doi.org/10.1371/journal.pone.0093565

Carey, S. (2001). Cognitive Foundations of Arithmetic: Evolution and Ontogenisis. Mind and Language, 16(1), 37-55. http://doi.org/10.1111/1468-0017.00155

Castronovo, J., \& Göbel, S. M. (2012). Impact of high mathematics education on the number sense. PloS One, 7(4), e33832. article. http://doi.org/10.1371/journal.pone.0033832

Cohen, D. J., \& Sarnecka, B. W. (2014). Children's Number-Line Estimation Shows Development of Measurement Skills (Not Number Representations). Developmental Psychology. http://doi.org/10.1037/a0035901

Condry, K. F., \& Spelke, E. S. (2008). The development of language and abstract concepts: the case of natural number. Journal of Experimental Psychology. General, 137(1), 22-38. article. http://doi.org/10.1037/0096-3445.137.1.22

Cutini, S., Scarpa, F., Scatturin, P., Dell'Acqua, R., \& Zorzi, M. (2014). Number-space interactions in the human parietal cortex: Enlightening the snarc effect with functional near-infrared spectroscopy. Cerebral Cortex, 24(2), 444-451. http://doi.org/10.1093/cercor/bhs321

Davidson, K., Eng, K., \& Barner, D. (2012). Does learning to count involve a semantic induction? 
Cognition, 123(1), 162-73. article. http://doi.org/10.1016/j.cognition.2011.12.013

de Hevia, M. D., \& Spelke, E. S. (2010). Number-Space Mapping in Human Infants. Psychological Science, 21(5), 653-660. http://doi.org/10.1177/0956797610366091

Ebersbach, M., Luwel, K., Frick, A., Onghena, P., \& Verschaffel, L. (2008). The relationship between the shape of the mental number line and familiarity with numbers in 5 - to 9-year old children: evidence for a segmented linear model. Journal of Experimental Child Psychology, 99(1), 1-17. http://doi.org/10.1016/j.jecp.2007.08.006

Field, A., Miles, J., \& Field, Z. (2012). Discovering Statistics Using R. Sage.

Fischer, U., Moeller, K., Bientzle, M., Cress, U., \& Nuerk, H.-C. (2011). Sensori-motor spatial training of number magnitude representation. Psychonomic Bulletin \& Review, 18(1), 177-83. http://doi.org/10.3758/s13423-010-0031-3

Geary, D. C., Hoard, M. K., Nugent, L., \& Byrd-Craven, J. (2008). Development of number line representations in children with mathematical learning disability. Developmental Neuropsychology, 33(3), 277-299. article. http://doi.org/10.1080/87565640801982361

Gelman, R., \& Gallistel, C. R. (1978). The Child's Understanding of Number. Educational Researcher (Vol. 8). Cambridge, MA: Harvard University Press.

Gobel, S. M., Shaki, S., \& Fischer, M. H. (2011). The Cultural Number Line: A Review of Cultural and Linguistic Influences on the Development of Number Processing. Journal of Cross-Cultural Psychology, 42(4), 543-565. http://doi.org/10.1177/0022022111406251

Gunderson, E. a., Spaepen, E., \& Levine, S. C. (2015). Approximate number word knowledge before the cardinal principle. Journal of Experimental Child Psychology, 130, 35-55. http://doi.org/10.1016/j.jecp.2014.09.008 
Hannula, M. M., \& Lehtinen, E. (2005). Spontaneous focusing on numerosity and mathematical skills of young children. Learning and Instruction, 15(3), 237-256. article. http://doi.org/10.1016/j.learninstruc.2005.04.005

Hannula, M. M., Lepola, J., \& Lehtinen, E. (2010). Spontaneous focusing on numerosity as a domain-specific predictor of arithmetical skills. Journal of Experimental Child Psychology, 107(4), 394-406. article. http://doi.org/10.1016/j.jecp.2010.06.004

Hubbard, E. M., Piazza, M., Pinel, P., \& Dehaene, S. (2005). Interactions between number and space in parietal cortex. Nature Reviews Neuroscience, 6(6), 435-448. article. http://doi.org/10.1038/nrn1684

Hurst, M., Leigh Monahan, K., Heller, E., \& Cordes, S. (2014). 123s and ABCs: developmental shifts in logarithmic-to-linear responding reflect fluency with sequence values. Developmental Science, 1-13. http://doi.org/10.1111/desc. 12165

Kramer, P., Stoianov, I., Umiltà, C., \& Zorzi, M. (2011). Interactions between perceptual and numerical space. Psychonomic Bulletin \& Review, 18(4), 722-728. http://doi.org/10.3758/s13423-011-0149-y

Landerl, K., Bevan, A., \& Butterworth, B. (2004). Developmental dyscalculia and basic numerical capacities: a study of 8-9-year-old students. Cognition, 93(2), 99-125. http://doi.org/10.1016/j.cognition.2003.11.004

Le Corre, M. (2014). Children acquire the later-greater principle after the cardinal principle. British Journal of Developmental Psychology, 32(2), 163-177. http://doi.org/10.1111/bjdp.12029

Le Corre, M., \& Carey, S. (2007). One, two, three, four, nothing more: an investigation of the conceptual sources of the verbal counting principles. Cognition, 105(2), 395-438. 
http://doi.org/10.1016/j.cognition.2006.10.005

Le Corre, M., Van de Walle, G., Brannon, E. M., \& Carey, S. (2006). Re-visiting the competence/performance debate in the acquisition of the counting principles. Cognitive Psychology, 52(2), 130-69. http://doi.org/10.1016/j.cogpsych.2005.07.002

Lee, M. D., \& Sarnecka, B. W. (2010). A Model of Knower-Level Behavior in Number-Concept Development. Cognitive Science, 34(1), 51-67. http://doi.org/10.1111/j.1551-6709.2009.01063.x

Lee, M. D., \& Sarnecka, B. W. (2011). Number-knower levels in young children: insights from Bayesian modeling. Cognition, 120(3), 391-402. http://doi.org/10.1016/j.cognition.2010.10.003

Lourenco, S. F., \& Longo, M. R. (2010). General magnitude representation in human infants. Psychological Science : A Journal of the American Psychological Society / APS, 21(6), 873-81. http://doi.org/10.1177/0956797610370158

McCrink, K., \& Opfer, J. E. (2014). Development of Spatial-Numerical Associations. Current Directions in Psychological Science, 23(6), 439-445. http://doi.org/10.1177/0963721414549751

Merkley, R., \& Ansari, D. (2016). Why numerical symbols count in the development of mathematical skills: evidence from brain and behavior. Current Opinion in Behavioral Sciences, 10, 14-20. http://doi.org/10.1016/j.cobeha.2016.04.006

Moeller, K., Pixner, S., Kaufmann, L., \& Nuerk, H.-C. (2009). Children's early mental number line: logarithmic or decomposed linear? Journal of Experimental Child Psychology, 103(4), 503-15. http://doi.org/10.1016/j.jecp.2009.02.006

Muldoon, K., Towse, J., Simms, V., Perra, O., \& Menzies, V. (2013). A longitudinal analysis of estimation, counting skills, and mathematical ability across the first school year. Developmental Psychology, 49(2), 250-7. http://doi.org/10.1037/a0028240 
Negen, J., Sarnecka, B. W., \& Lee, M. D. (2012). An Excel sheet for inferring children's number-knower levels from give-N data. Behavior Research Methods, 44(1), 57-66. http://doi.org/10.3758/s13428-011-0134-4

Nunez, R., \& Fias, W. (2015). Ancestral Mental Number Lines: What Is the Evidence? Cognitive Science, 1-5. http://doi.org/10.1111/cogs.12296

Odic, D., Le Corre, M., \& Halberda, J. (2015). Children's mappings between number words and the approximate number system. Cognition, 138, 102-121. http://doi.org/10.1016/j.cognition.2015.01.008

Opfer, J. E., Thompson, C. a, \& Furlong, E. E. (2010). Early development of spatial-numeric associations: evidence from spatial and quantitative performance of preschoolers. Developmental Science, 13(5), 761-71. article. http://doi.org/10.1111/j.1467-7687.2009.00934.x

Patro, K., \& Haman, M. (2012). The spatial-numerical congruity effect in preschoolers. Journal of Experimental Child Psychology, 111(3), 534-42. http://doi.org/10.1016/j.jecp.2011.09.006

Ramani, G. B., Siegler, R. S., \& Hitti, A. (2012). Taking it to the classroom: Number board games as a small group learning activity. Journal of Educational Psychology, 104(3), 661-672. http://doi.org/10.1037/a0028995

Ranzini, M., Lisi, M., Blini, E., Pitteri, M., Treccani, B., Priftis, K., \& Zorzi, M. (2014). Larger, smaller, odd or even? Task-specific effects of optokinetic stimulation on the mental number space. Journal of Cognitive Psychology, 5911(August), 1-12. http://doi.org/10.1080/20445911.2014.941847

Ranzini, M., Lisi, M., \& Zorzi, M. (2016). Voluntary eye movements direct attention on the mental number space. Psychological Research, 80(3), 389-398. 
http://doi.org/10.1007/s00426-015-0741-2

Sarnecka, B. W., \& Carey, S. (2008). How counting represents number: what children must learn and when they learn it. Cognition, 108(3), 662-74. http://doi.org/10.1016/j.cognition.2008.05.007

Sarnecka, B. W., \& Gelman, S. A. (2004). Six does not just mean a lot: preschoolers see number words as specific. Cognition, 92(3), 1-23. http://doi.org/10.1016/j.cognition.2003.10.001.Six

Sasanguie, D., De Smedt, B., Defever, E., \& Reynvoet, B. (2012). Association between basic numerical abilities and mathematics achievement. British Journal of Developmental Psychology, 30(2), 344357. http://doi.org/10.1111/j.2044-835X.2011.02048.x

Sasanguie, D., Göbel, S. M., Moll, K., Smets, K., \& Reynvoet, B. (2013). Approximate number sense, symbolic number processing, or number-space mappings: what underlies mathematics achievement? Journal of Experimental Child Psychology, 114(3), 418-31. http://doi.org/10.1016/j.jecp.2012.10.012

Sasanguie, D., Van den Bussche, E., \& Reynvoet, B. (2012). Predictors for Mathematics Achievement? Evidence From a Longitudinal Study. Mind, Brain, and Education, 6(3), 119-128. http://doi.org/10.1111/j.1751-228X.2012.01147.x

Sella, F., Berteletti, I., Brazzolotto, M., Lucangeli, D., \& Zorzi, M. (2014). Number line estimation in children with developmental dyscalculia. Learning Disabilties: A Contemporary Journal, 11(2), $41-49$.

Sella, F., Berteletti, I., Lucangeli, D., \& Zorzi, M. (2015a). Spontaneous non-verbal counting in toddlers. Developmental Science, 1-9. http://doi.org/10.1111/desc.12299

Sella, F., Berteletti, I., Lucangeli, D., \& Zorzi, M. (2015b). Varieties of Quantity Estimation in Children. Developmental Psychology, 51(6), 758-770. http://doi.org/10.1037/a0039183 
Sella, F., Sader, E., Lolliot, S., \& Cohen Kadosh, R. (2016). Basic and advanced numerical performances relate to mathematical expertise but are fully mediated by visuospatial skills. Journal of Experimental Psychology: Learning, Memory, and Cognition.

Siegler, R. S., \& Booth, J. L. (2004). Development of numerical estimation in young children. Child Development, 75(2), 428-44. article. http://doi.org/10.1111/j.1467-8624.2004.00684.x

Siegler, R. S., \& Lortie-Forgues, H. (2014). An Integrative Theory of Numerical Development. Child Development Perspectives, O(0), n/a-n/a. http://doi.org/10.1111/cdep.12077

Siegler, R. S., \& Opfer, J. E. (2003). The development of numerical estimation: evidence for multiple representations of numerical quantity. Psychological Science, 14(3), 237-43. article.

Siegler, R. S., \& Ramani, G. B. (2008). Playing linear numerical board games promotes low-income children's numerical development. Developmental Science, 11(5), 655-61. http://doi.org/10.1111/j.1467-7687.2008.00714.x

Siegler, R. S., \& Ramani, G. B. (2009). Playing linear number board games - but not circular ones improves low-income preschoolers' numerical understanding. Journal of Educational Psychology, 101(3), 545-560. http://doi.org/10.1037/a0014239

Siegler, R. S., Thompson, C. a., \& Opfer, J. E. (2009). The Logarithmic-To-Linear Shift: One Learning Sequence, Many Tasks, Many Time Scales. Mind, Brain, and Education, 3(3), 143-150. http://doi.org/10.1111/j.1751-228X.2009.01064.x

Simms, V., Clayton, S., Cragg, L., Gilmore, C., \& Johnson, S. (2016). Explaining the relationship between number line estimation and mathematical achievement: The role of visuomotor integration and visuospatial skills. Journal of Experimental Child Psychology, 145, 22-33. http://doi.org/10.1016/j.jecp.2015.12.004 
Slusser, E. B., Santiago, R. T., \& Barth, H. C. (2013). Developmental change in numerical estimation. Journal of Experimental Psychology. General, 142(1), 193-208. http://doi.org/10.1037/a0028560

Stevenson, A., \& Lindberg, C. A. (2010). New Oxford American dictionary. (3rd ed.). New York: Oxford University Press.

Stoianov, I., Kramer, P., Umiltà, C., \& Zorzi, M. (2008). Visuospatial priming of the mental number line. Cognition, 106(2), 770-779. http://doi.org/10.1016/j.cognition.2007.04.013

Sullivan, J., \& Barner, D. (2014). Inference and Association in Children's Early Numerical Estimation. Child Development, 1-16. http://doi.org/10.1111/cdev.12211

Thompson, J. M., Nuerk, H. C., Moeller, K., \& Cohen Kadosh, R. (2013). The link between mental rotation ability and basic numerical representations. Acta Psychologica, 144(2), 324-331. http://doi.org/10.1016/j.actpsy.2013.05.009

Verguts, T., Fias, W., \& Stevens, M. (2005). A model of exact small-number representation. Psychonomic Bulletin \& Review, 12(1), 66-80. article. Retrieved from http://www.ncbi.nlm.nih.gov/pubmed/15945201

Vuilleumier, P., Ortigue, S., \& Brugger, P. (2004). The number space and neglect. Cortex; a Journal Devoted to the Study of the Nervous System and Behavior, 40(2), 399-410. http://doi.org/10.1016/S0010-9452(08)70134-5

Wiese, H. (2003). Iconic and non-iconic stages in number development: The role of language. Trends in Cognitive Sciences, 7(9), 385-390. http://doi.org/10.1016/S1364-6613(03)00192-X

Wynn, K. (1990). Children's understanding of counting. Cognition, 36, 155-193.

Wynn, K. (1992). Children's Acquisition of the Number Words and the Counting System. Cognitive Psychology, 24(24), 220-251. 
Young, C. J., Marciani, F. E., \& Opfer, J. E. (2011). Linear Numerical Magnitude Representations Aid Memory for Single Numbers. In Proceedings of the XXXIII Annual Conference of the Cognitive Science Society. Mahwah, NJ: Erlbaum. (pp. 1509-1514).

Zorzi, M., Bonato, M., Treccani, B., Scalambrin, G., Marenzi, R., \& Priftis, K. (2012). Neglect Impairs Explicit Processing of the Mental Number Line. Frontiers in Human Neuroscience, 6(May), 1-12. http://doi.org/10.3389/fnhum.2012.00125

Zorzi, M., \& Butterworth, B. (1999). A computational model of number comparison. In M. Hahn \& S. Stoness (Eds.), Twenty First Annual Meeting of the Cognitive Science Society (p. 1). Mahwah, NJ.: Mahwah.

Zorzi, M., Priftis, K., \& Umiltà, C. (2002). Neglect disrupts the mental number line. Nature, 417(May), 138-140.

Zorzi, M., Stoianov, I., \& Umiltà, C. (2005). Computational Modeling of Numerical Cognition. In Handbook of mathematical cognition (Vol. 19, pp. 67-84). 